\title{
Characterization of flow transport within pore spaces of an open-work gravel bed
}

\author{
Susithra Lakshmanan ${ }^{1,3 *}$, Gareth Pender ${ }^{1}$, Heather Haynes ${ }^{1}$, William Holmes ${ }^{2}$ \\ ${ }^{1}$ Institute for Infrastructure and Environment, Heriot-Watt University, Edinburgh, United Kingdom \\ ${ }^{2}$ Glasgow Experimental MRI Centre, Institute of Neuroscience and Psychology, University of Glasgow, United Kingdom \\ ${ }^{3}$ Department of Chemical Engineering \& Biotechnology, University of Cambridge, United Kingdom \\ *Corresponding author E-mail: susithra02@gmail.com
}

Copyright $($ ) 2014 Susithra Lakshmanan et al. This is an open access article distributed under the Creative Commons Attribution License, which permits unrestricted use, distribution, and reproduction in any medium, provided the original work is properly cited.

\begin{abstract}
Analysis of the flow dynamics within the near-bed and sub-surface regions of river bed sediment is critical in understanding fluid exchange and related chemical transfer/reactions. The knowledge in above is limited as these regions are difficult to measure using traditional instrumentation methods. In this paper, we tried the use of Magnetic Resonance Imaging (MRI) technique to non-invasively image flow dynamics of simulated river bed. We developed a bespoke MRI-compatible open-channel flume in order to acquire real-time flow images from within the MRI bore and used contrast agent (Gd-DTPA) as a tracer through an immobile, porous gravel bed. Single MR Image slices along the flume length were obtained for analysis. The flow tracer images from within the sediment bed are calibrated from the output data in order to provide fully quantitative maps of tracer concentration at regular time intervals. These 'whitebox' (i.e. data from within the porous bed) tracer profiles were evaluated with the CXTFIT computer package to estimate the transport parameters. The intention was both, to illustrate the appropriateness of MRI for flow-sediment research and to analyse the relationship between tracer dispersion and gravel framework structure.
\end{abstract}

Keywords: Porous Gravel Bed, Magnetic Resonance Imaging, Tracer Transport, CXTFIT.

\section{Introduction}

Magnetic resonance imaging (MRI) technique has the ability to collect spatio-temporal data of flow within opaque, porous systems. The added advantage is its non-invasiveness and it does not hinder or alter the flow. These characteristics helps in overcoming many present limitations of many techniques (e.g. Particle Imaging Velocimetry) and provides immense scope for detailed analysis of both sub-surface flow-related processes and analysis of the surface-subsurface interactions and the sediment boundary for open-flow research. Despite very limited evidence of the use of MRI by river-based researchers, the benefits of this technique have been long since exploited in wider flowsediment disciplines with examples including: sedimentary structure in sandstone cores [1], acquiring 3D bed structure of sand-gravel sediments [2], hydro-carbon bearing in sedimentary rocks for the oil and gas industry [3] and mapping of flow velocity through porous media [4].

Thus, the present paper seeks to develop an appropriate equipment and methodology that suited for two purposes; (i) imaging open-channel flow with focus of interest on surface and subsurface regions, and (ii) undertaking quantitative image analysis appropriate to ascertaining relationships between flow processes and open-pore framework structure. Specifically, this study employs a GD-DTPA tracer (complex of gadolinium paramagnetic metal) to image water flow above and within a gravel bed screeded inside the flume. This method helps to acquire comparatively high quality signals during imaging as the relaxation of the $1 \mathrm{H}$ (hydrogen) nuclei surrounded by the paramagnetic molecules create sufficient contrast for the imaging process. Calibrating these images provides concentration profiles which are then used to predict the transport parameters of the tracer as a proxy for flow processes. CXTFIT a computer program is widely used to estimate the transport parameters in a laboratory or field data sets under various conditions. This program is used in the present study to estimate the tracer dispersion at various time intervals. 


\section{Materials and Methodology}

\subsection{Open-Channel Flume}

In order to image spatio-temporal flow dynamics, this project required the design and construction of a bespoke MRcompatible open channel flume with the capability of variable discharge, fine sediment feed and easy to construct/dismantle within the MR facility. As magnetic materials would be attracted to the internal magnet and coils of the MRI, all flume components had to be made of Perspex and all the fixings made watertight via rubber seals. A new flume with above characteristics was fabricated.

This flume set up was tested in the 7Tesla GEMRIC MRI facility of The University of Glasgow, UK (Fig. 1). In all other ways, the flume design was a traditional open-channel set-up with rectangular cross-section with size of $9.3 \mathrm{~cm}$ width $\times 6.2 \mathrm{~cm}$ depth $\times 3 \mathrm{~m}$ length. The above dimensions were approximated to make a largest possible flume that fit within the bore of the MRI $(152 \mathrm{~mm})$. Due to the metal components that associated with the pump, this was kept outside the MRI room with plastic pipe connections running through the wall between the pump and the MRI rooms. The slope of the flume was established by adjusting the screws that are holding the flume within the bore of the MRI system and the slope of $1 \mathrm{in} 200$ was used for all the experiments. The flow of water was established with a flow rate of 0.1 lit/sec. A feeder tank with agitation system is connected to the inflow pipe as appropriate for dispersal of commercially available paramagnetic Gd-DTPA (Sigma Aldrich) contrast agent and this had a manual top-up valve to feed the tracer throughout the experiment. As part of the main experimental programme, a gravel bed (4-5mm diameter dolomite) was screeded flat to a depth of $36 \mathrm{~mm}$ within the flume test section; Fig. 2 shows the overall MR-compatible flume set-up used for this experiment.

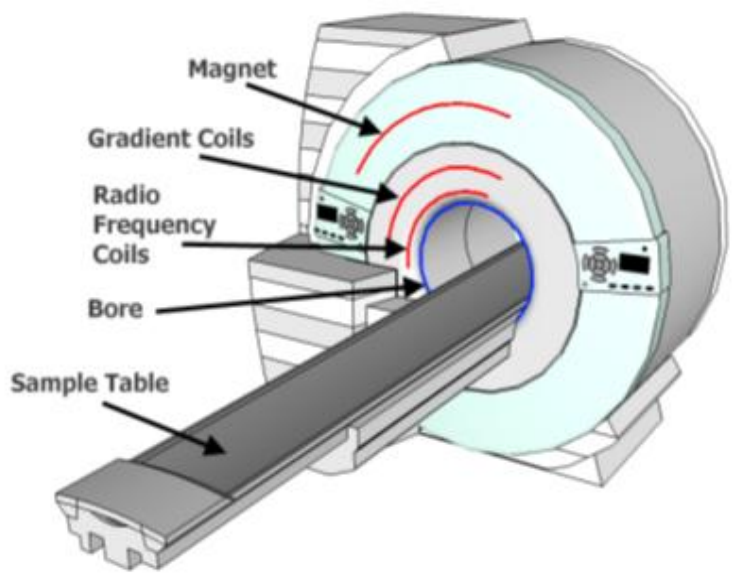

Fig. 1: Schematic Image of an MRI, Indicating the 360 Degree Arrangement of the Magnetic and Location of the Bore (152mm Diameter) Used To Hold the Flume

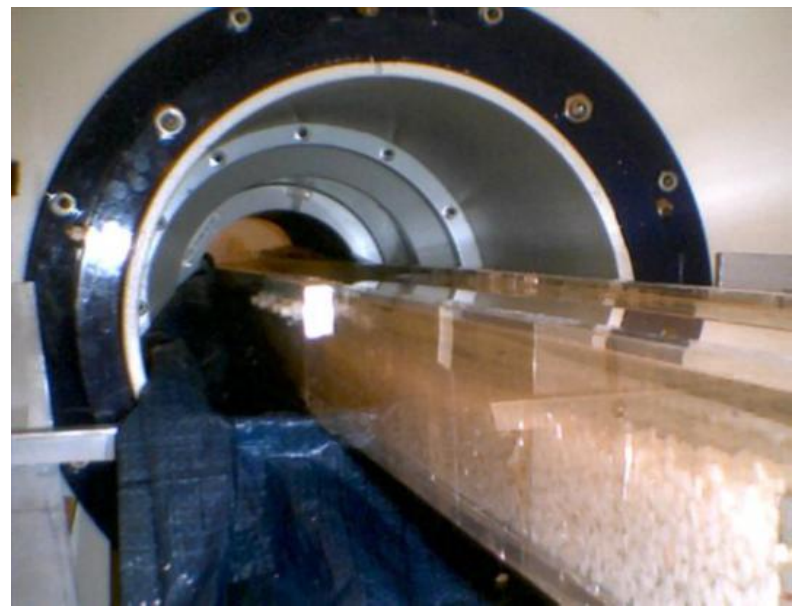

Fig. 2: Photograph of the MR- Compatible Open-Channel Flume within the Bore of MRI System, Including the Dolomite Gravel Bed. 


\subsection{Magnetic Resonance Imaging}

The MRI experiments were performed on a Bruker Avance BioSpec system with 7T superconducting magnet (Bruker BioSpin, Karlsruhe, Germany). Pilot scans indicated that dolomite lithology provided the best MR image quality due to low ferromagnetic and paramagnetic impurities which was in agreement with previous research [2], [5]. Hence it was used within the flume set-up shown in Fig. 2. This flume was placed inside MRI magnet bore bed horizontally with above mentioned tilt gradient for the flume (Fig. 1). Uniform flow was established at a flow depth 10-20mm and rate of $<11 / \mathrm{s}$. The $1 \mathrm{H}$ magnetic nuclei of the water were used as the imaging medium for the MRI.

Tracer transport imaging was performed using a Rapid Acquisition Relaxation Enhanced (RARE) Sequence (see Section 2.3) along a single vertical slice taken along the centreline of the length of the flume. The field of view of the image was $250 \mathrm{~mm}$ (length) x $125 \mathrm{~mm}$ (depth). The movement of the tracer through the system was imaged at a five minute repeat interval and post-processed to provide fully quantitative maps of tracer concentrations.

\subsection{Calibration of GD-DTPA}

The effect of tracer concentration on the relaxation times is represented by the following equation [6], [7]

$$
[C]=\frac{1}{R}\left[\frac{1}{T_{2, i}}-\frac{1}{T_{2,0}}\right]
$$

Where $T_{2,0}$ is the relaxation time in the absence of paramagnetic tracer, $T_{2, i}$ is the relaxation time in the presence of paramagnetic tracer, [C] is the concentration of the paramagnetic tracer, and $R$ is the relaxivity constant of the paramagnetic tracer. To determine the relaxivity constant R, standards of different Gd -DTPA concentrations were prepared and their $T_{2}$ values measured with a multiple spin-echo sequence.

\subsection{Quantitative Measurement of Tracer Concentration}

Whilst a detailed review of the physics of MRI can be found in Levitt [8], it is pertinent to outline the basics of the technique here. The signal decay at a particular concentration $C$ is obtained using a Rapid Acquisition Relaxation Enhancement pulse sequence (RARE) and it is represented by [9];

$$
\frac{S}{S_{0}}=\exp \left(\frac{-t_{e}}{T_{2, i}}\right)
$$

Where $\mathrm{S}$ is the MRI signal, $\mathrm{S} 0$ is the signal at zero echo time and $\mathrm{T}_{2}$ is the spin-spin relaxation time. The effective echo time $t_{e}$ was chosen to maximize the signal contrast as described in von der Schulenburg et al [10] and was determined to be $170 \mathrm{~ms}$. Combining equations (1) and (2) gives the following equation:

$$
C=\left[\left(\ln \left(\frac{S}{S_{0}}\right) /-t_{e}\right)-\left(\frac{1}{T_{2,0}}\right)\right] \frac{1}{R}
$$

A RARE image of the sample containing only water was appropriately scaled, to get a maximum signal intensity map of $S_{0}$ Hence, GD tracer concentrations can be calculated from equation (3), using $T_{2}$ weighted images acquired before and after introduction of the tracer.

\subsection{CXTFIT Modelling}

CXTFIT model is widely used to estimate the transport parameters in surface and sub-surface flow applications [11]. The hydrodynamic dispersion coefficient is estimated using the following analytic solutions of the linear convectiondispersion equation (CDE) with a first-order loss term [12] in the CXTFIT- Excel package.

$$
\frac{\partial}{\partial t} C=D \frac{\partial^{2} C}{\partial x^{2}}-v_{p} \frac{\partial C}{\partial x}-k C
$$

Where $\mathrm{C}$ is the concentration, $t$ is time, $x$ is distance from the inlet, $D$ is the dispersion coefficient, $v_{P}$ is average particle velocity and $k$ is the deposition rate coefficient. The flow chart of CXTFIT analysis is shown in Fig. 3 and the full details of setting up the model including parameters estimation and optimization sequence are discussed in Tang et al [12] and Wraith et al [13]. The input parameters are the column length, average velocity and pulse duration and the model parameters are dispersivity, mobile water fraction $\beta$ and mass transfer coefficient $\omega$. 


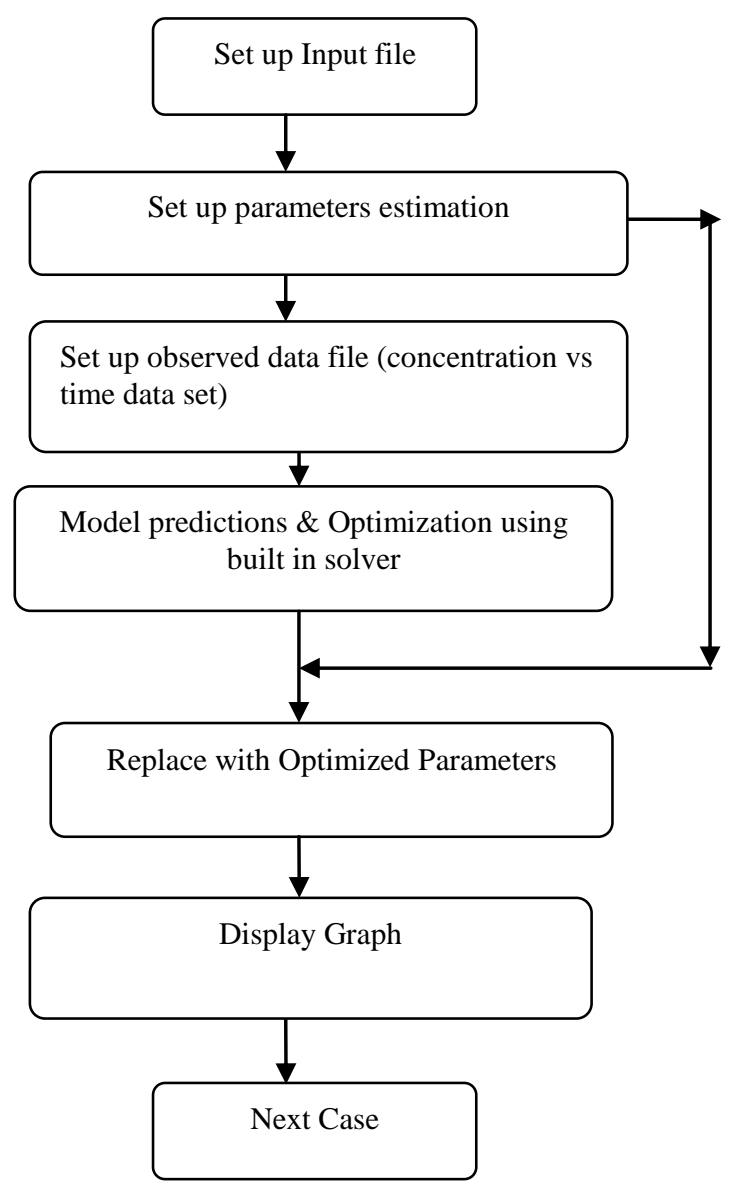

Fig. 3: Flow Chart of the CXTFIT Analysis

These parameters give the best-fit, measured by the least-square error between modelled and observed concentration profiles. From dispersivity, the dispersion coefficient $D$ is calculated over various time points. The deposition rate coefficient $k$ can be calculated from the other parameters $v_{P}, \beta$ and $\omega$.

\section{Results and Discussion}

\subsection{Relaxivity Constant Measurement}

Various tracer concentrations in water were prepared to calculate the relaxivity constant. The relationship between $\mathrm{T}_{2}$ and the tracer concentration is shown in Fig.4.

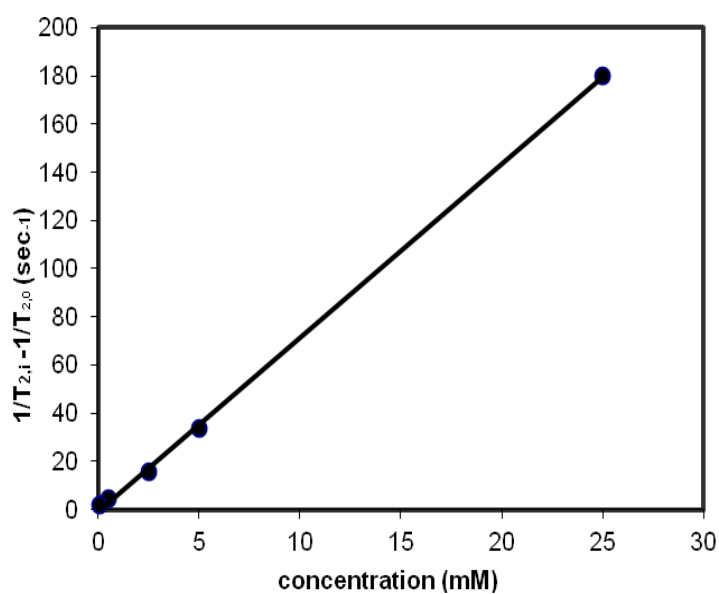

Fig. 4: Relaxation Rate with Respect to GD DTPA Concentration 
The Relaxivity constant, $\mathrm{R}$, is the slope of the line in the above graph and was determined by the least square fit method. This was calculated as $7.2 \mathrm{mM}^{-1} \mathrm{~s}^{-1}$. This constant was then applied in equation (3) to analyze the tracer concentration profiles throughout the bed experiments. The analysis was done by using IDL image processing software.

\subsection{Transport Study of GD-DTPA through the Sediment bed}

Spatially and temporally resolved $T_{2}$ - weighted images of the tracer transport through the sediment bed were acquire for a single slice along the centreline of the flume at 5 minute time intervals until the image was saturated. Selected time intervals are shown in Fig. 5 (a) to (d).

(A)

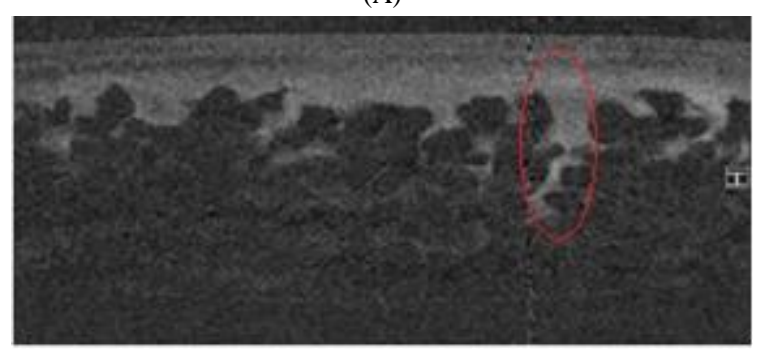

(C)

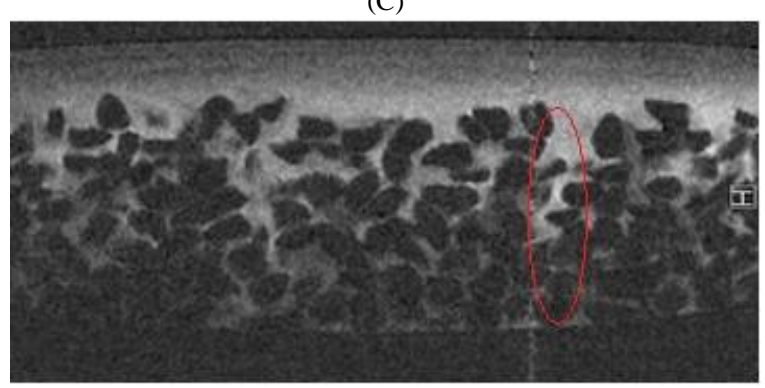

(B)

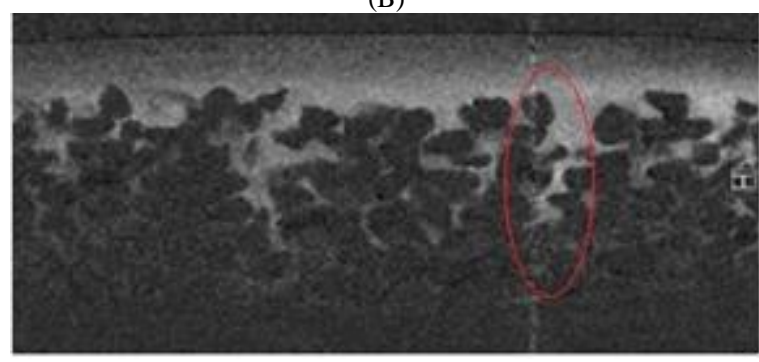

(D)

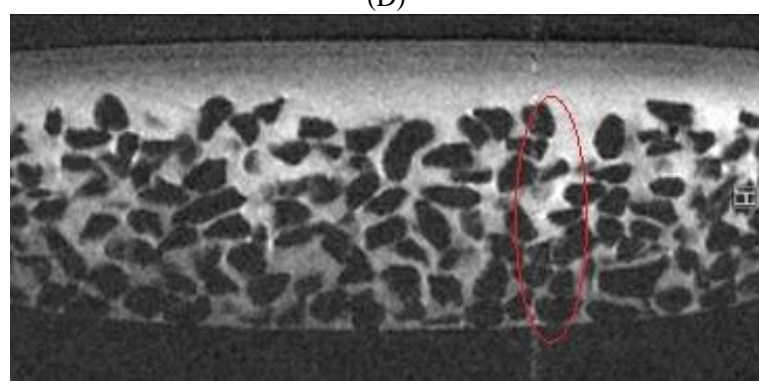

Fig. 5: $T_{2}$ Weighted Images of GD DTPA in Dolomite Gravel at Time (A) 15, (B) 20, (C) 25, and (D) 40 Minutes; GD Transport Shown by Bright Regions. Rapid Penetration of Flow Through a Wide and Well-Connected Surface Pore Is Highlighted In Red.

The brightest regions of the image are directly related to Gd concentration. Thus, two findings are clearly evident. Firstly, tracer penetration of the bed is faster in locations where the surface pores are larger and pore throat connections are wider (Fig. 5); this permits faster transfer of tracer fluid to the lower gravel layers. The highlighted location on Fig. 5 specifically shows a large surface pore of diameter $4-5 \mathrm{~mm}$ and connected pore throats $>1 \mathrm{~mm}$ diameter for 4-5 layers of gravel. Thus, a tracer concentration (image brightness) is equivalent to that of the above-surface flow field, indicating a free exchange of fluid flow throughout this depth of bed at this location. The implication is that strong vertical velocities exist in this location and that this region of bed.

Secondly, Fig. 5 clearly indicates a separate, slower throughflow process within the subsurface. After 15 minutes the majority of the bed indicates no Gd penetration. Progressive increases in Gd tracer occur over the next 25 minutes, but it takes 40 minutes until the concentration of the pore fluid is equivalent to that of the surface free stream. Thus, comparing throughflow to surface-penetration data suggests that through flow is $\sim 3$ times slower at tracer dispersal than penetrating flow.

\subsection{Tracer Concentration Profiles}

Using the above discussed calibration protocol in section 2; these $T_{2}$ weighted images are converted into actual concentration maps of Gd DTPA. Averaged Gd concentrations across the length of the image slice from $15 \mathrm{~min}$ at 5 minute time intervals are shown in Fig.6. 

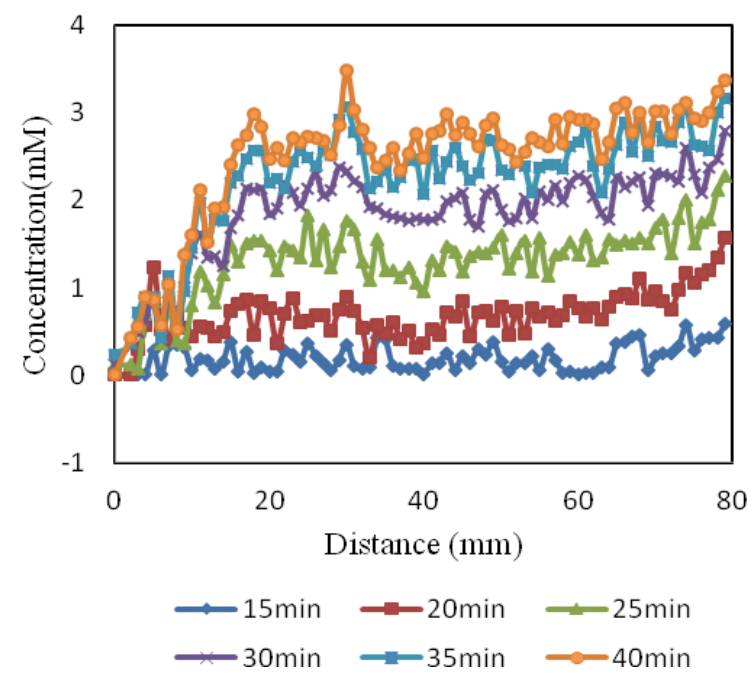

Fig. 6: Averaged Concentration Profiles of GD DTPA Concentration from 15 to 40 Minutes.

As we have seen from Fig. 5 (a) there is not much tracer penetration at $15 \mathrm{~min}$ and starting to fill in the pore spaces and this progressive increase can be clearly seen in the concentration profiles in Fig.6.

\subsection{Determination of Dispersivity - CXTFIT}

The experimental results were evaluated with CXTFIT to quantify the dispersion coefficient. The more saturated profiles achieved goodness of fit values $\left(\mathrm{R}^{2}\right) 77 \%$, 79\%, 86\% for 30-40mins and the profiles are shown in Fig.7 (a) to (c). The model fit for earlier time points have less saturated concentration profiles and hence did not give reliable fit. The fitted parameters are listed in Table 1.

(A)

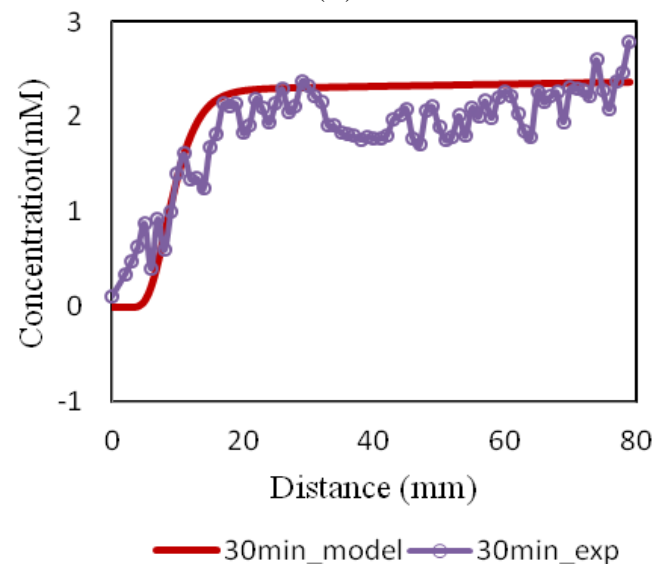

(B)

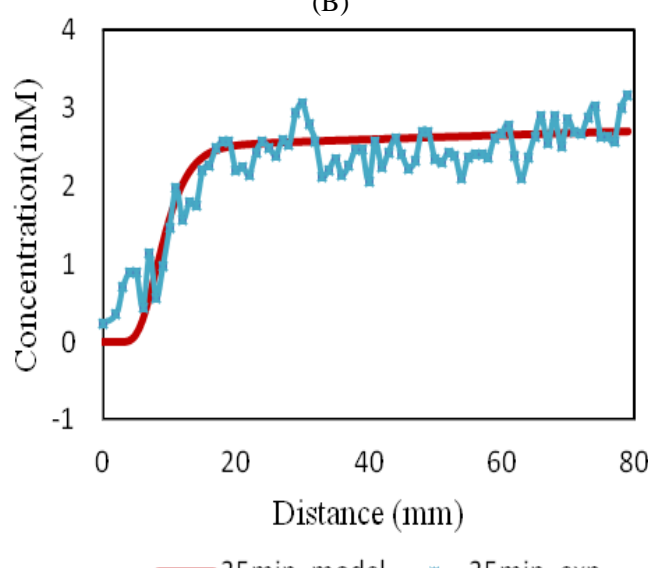

(C)

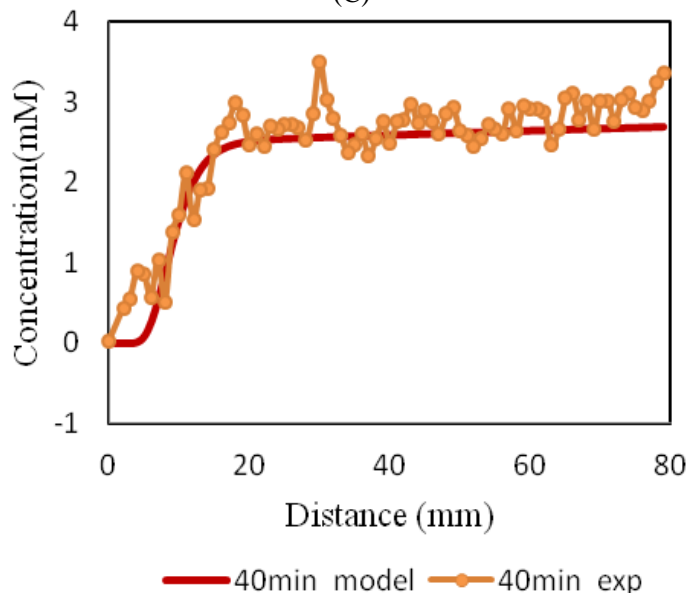

Fig. 7: Experimental Vs. Cxtfit Simulated Tracer Profiles For (A) 30, (B) 35 and (C) 40 Min 
Table 1: Fitted Parameters from CXTFIT

\begin{tabular}{ccccc}
\hline Time $(\mathrm{min})$ & $\mathrm{D}\left(\mathrm{m}^{2} / \mathrm{s}\right)$ & $\beta$ & $\omega$ & $\mathrm{R}^{2}(\%)$ \\
\hline 30 & $2.7107 \times 10^{-06}$ & 0.125 & 0.30 & 77 \\
35 & $2.7033 \times 10^{-06}$ & 0.125 & 0.32 & 79 \\
40 & $2.722 \times 10^{-06}$ & 0.125 & 0.39 & 86 \\
\hline
\end{tabular}

As it is shown in the table, the achieved dispersion coefficient is $2.7 \times 10^{-06} \mathrm{~m}^{2} / \mathrm{s}$. There is no similar study found in the literature. However, using nonreactive tracer through soil columns Supriya et al [14] achieved the dispersion coefficient of $5.67 \times 10^{-10}-9.45 \times 10^{-09} \mathrm{~m}^{2} / \mathrm{s}$ for varying the bed height and Disli [15] obtained the dispersion coefficient of $5.35 \times 10^{-}$ ${ }^{10}-2.08 \times 10^{-07} \mathrm{~m}^{2} / \mathrm{s}$ for various soil types using CXTFIT. The variations in the dispersivity estimation could be due to influence of soil types, bed structure/ the packing arrangements and bed height. The other fitted parameters $\beta$ and $\omega$ can be used in analyzing the mobility and retention of tracer in the system if the tracer has any surface charge or any reactive conditions. This will give further details about deposition rate and the attachment/detachment efficiency of the tracer to the sediment.

\section{Conclusion}

This paper has designed and utilized a pioneering coupled flume-MRI set-up to provide images of flow within a porous sediment bed. The data acquired demonstrated a tracer-transport proxy for imaging the fluid dynamics of the bed subsurface and the surface-subsurface interaction. Whilst the present study is restricted to a single $2 \mathrm{D}$ image slice, the methodology is appropriate to multiple $2 \mathrm{D}$ imaging or even $3 \mathrm{D}$ volumetric imaging of the flow field and porous structure. Such non-invasive methodology therefore warrants further investigation for quantitative analysis of a wider range of flow-sediment processes in the near-bed and sub-surface regions of open-channel flow systems. CXTFIT is used to quantify the dispersion coefficient and this can be applied in real life situations for evaluating the environmental impacts of pollutant transport in natural and engineered coarse grained systems, such as sustainable urban drainage systems and river-beds.

\section{Acknowledgements}

This research was undertaken with financial support from the Larger Grants scheme of The Carnegie Trust for the Universities of Scotland, as held by the third author. The authors would also like to express their sincere thanks to Jim Mullen and Stuart Mclean for their assistance with the flume set up and MRI experiments.

\section{References}

[1] Marica,F., Chen, Q., Hamilton, A., Hall, C., Al, T., Balcom, B., Spatially resolved measurement of rock core porosity. Journal of Magnetic Resonance, vol.178, 2006, 136-141. http://dx.doi.org/10.1016/j.jmr.2005.09.003.

[2] Haynes, H., Vignaga, E and Holmes, W.M., Using magnetic resonance imaging for experimental analysis of fine-sediment infiltration into gravel beds. Sedimentology. vol.56, 2009, 1961-1975. http://dx.doi.org/10.1111/j.1365-3091.2009.01064.x.

[3] Johns, M.L. and Gladden, L.F. Surface-to-volume ratio of ganglia trapped in small-pore systems determined by pulsed-filed gradient nuclear magnetic resonance. J. Colloid.Interface Sci, vol.238, 2001, 96-104. http://dx.doi.org/10.1006/jcis.2001.7494.

[4] Baumann, T., Petsch, R., Niessner, R., Direct 3-D measurement of the flow velocity in porous media using magnetic resonance tomography. Environmental Science and Technology, vol.34, 2000, 4242-4248. http://dx.doi.org/10.1021/es991124i.

[5] Haynes, H., Ockelford A-M, Vignaga. E., Holmes, WM, A new approach to define surface/sub-surface transition in gravel beds. Acta Geophysica. Vol.60, 2012, 1589-1606. http://dx.doi.org/10.2478/s11600-012-0067-z.

[6] Bloembergen, N., Proton relaxation times in paramagnetic solutions. J. Chem. Phys, vol. 27, $1957, \quad 572-573$. http://dx.doi.org/10.1063/1.1743771.

[7] Phoenix, V.R., Holmes, W.M., Magnetic resonance imaging of structure, diffusivity and copper immobilization in a phototrophic biofilm. Appl. Environ. Microbiol, vol.74, 2008, 4934-4943. http://dx.doi.org/10.1128/AEM.02783-07.

[8] Levitt, MH, Spin Dynamics. John Wiley\&Sons: England, 2002.

[9] Haacke, E.M., Brown, R.W., Thompson, M.R., Venkatesan, R., Magnetic resonance imaging: Physical Principles and Sequence Design, Wiley Liss, 1999.

[10] Von der Schulenburg, D.A.G., Holland, D.J., Paterson Beedle, M., Macaskie, L.E., Gladden, L.F., Johns, M.L., Spatially resolved quantification of metal ion concentration in a biofilm-mediated ion exchanger. Biotechnology and Bioengineering, vol.99, 2008, 821-829. http://dx.doi.org/10.1002/bit.21647.

[11] Toride N, Leij FJ, Van Genuchten MT, The CXTFIT code for estimating transport parameters from laboratory or field tracer experiments, version 2.1. U.S. Salinity Laboratory. Agricultural Research Service US Department of Agriculture, Riverside, 1999.

[12] Tang, G., Mayes, M.A., Parker, J.C., Jardine, P.M. CXTFIT/Excel-A modular adaptable code for parameter estimation, sensitivity analysis and uncertainty analysis for laboratory or field tracer experiments. Computers \& Geosciences, vol.136, 2010, 1200-1209.

[13] Wraith, J.M., Or, D., Nonlinear parameter estimation using spreadsheet software. Journal of Natural Resources and Life Sciences Education. vol.27, 1998, 13-19.

[14] Supriya Pal, Somnath Mukherjee and Sudipta Ghosh, Application of Hydrus 1D model for assessment of phenol-soil adsorption dynamics, Environ. Sci. Pollut. Res, 21, 2014, 5249-5261. http://dx.doi.org/10.1007/s11356-013-2467-2.

[15] Disli E, Batch and column experiments to support heavy metals $(\mathrm{Cu}, \mathrm{Zn}$ and $\mathrm{Mn})$ in alluvial sediments. Ground Water Monitoring \& Remediation, vol. 30, no. 3, 2010, 125-139. http://dx.doi.org/10.1111/j.1745-6592.2010.01302.x. 